\title{
Frühe Krankengymnastik hat nur einen marginalen Effekt
}

Fragestellung: Ist eine früh einsetzende Krankengymnastik bei Patienten mit neu aufgetretenen lumbalen Rückenschmerzen wirksam?

Hintergrund: Die Lebenszeitprävalenz von akuten Rückenschmerzen beträgt $70 \%$ und $25 \%$ aller Erwachsenen berichten über Rückenschmerzen in den letzten drei Monaten. Rückenschmerzen sind für etwa 3-5\% aller Arztbesuche verantwortlich. Die meisten Patienten werden vom Hausarzt behandelt. Bei chronischen Rückenschmerzen ist der Nutzen von Krankengymnastik und Physiotherapie belegt. Für die Akutsituation gibt es bisher relativ wenige Daten.

Patienten und Methodik: Es handelt sich um eine randomisierte klinische Studie mit 220 Teilnehmern. Einschlusskriterien waren akute Rückenschmerzen in den letzten 16 Tagen, Alter zwischen 18 und 60 Jahre und ein Wert auf der Oswestry Disability Index Skala von 20 oder höher. Patienten mit radikulären Schmerzen wurden ausge-

Fritz JM, Magel JS, McFadden

$M$ et al. Early physical therapy vs usual care in patients with recent-onset low back pain: A randomized clinical trial. JAMA 2015; 314: 1459-67 schlossen. Alle Patienten erhielten Instruktionen wie sie sich bezüglich der Rückenschmerzen verhalten sollten. Bei der Hälfte der Patienten wurden vier Sitzungen einer Physiotherapie durchgeführt.
Diese begann üblicherweise 72 Stunden nach dem Erstkontakt mit dem Arzt. Die Physiotherapie erfasste unter anderem Manualtherapie und Bewegungsübungen. Außerdem wurden den Patienten Übungen zur Kräftigung der Muskulatur gezeigt.

Der primäre Outcome war die Veränderung auf der Ostwestry Disability Index Skala nach vier Wochen und nach einem Jahr. Außerdem wurden die Reduktion der Schmerzintensität, die Lebensqualität und die Inanspruchnahme des Gesundheitssystems untersucht. In die übliche Therapie wurden 112 Patienten randomisiert und in die frühe Physiotherapie 108. Die Patienten waren im Mittel 36 Jahre alt und $50 \%$ waren Frauen. Zwei Drittel der Patienten nahmen nicht steroidale Antirheumatika (NSAR) und ein Drittel Opioide ein. Zwei Drittel der Patienten hatten in der Vergangenheit unter Rückenschmerzen gelitten. Die mittlere Schmerzintensität auf einer Skala von 0 bis 10 wurde mit 5 angegeben. Der Wert auf der Oswestry Disability Index Skala lag bei 40. Nach drei Monaten fand sich ein signifikanter Unterschied zugunsten der Physiotherapie für die allgemeine Behinderung und nach vier Wochen für den Oswestry Disability Index Score. Unterschiede in der Schmerzintensität ließen sich nicht nachweisen. Die Inanspruchnahme des Gesundheitssystems war ebenfalls nicht unterschiedlich.

Schlussfolgerungen: Eine frühe Physiotherapie bei Patienten mit akuten lumbalen Rückenschmerzen führt zu einer geringen Verbesserung der Behinderung und der Schmerzintensität.

\section{- Kommentar von Hans-Christoph Diener, Essen}

\section{Erstmal abwarten und NSAR}

Diese prospektive randomisierte Studie zeigt, dass der frühe Einsatz einer Physiotherapie bei Patienten mit akuten Rückenschmerzen zu einer geringeren Besserung von Beschwerden und Behinderungen nach vier Wochen führt. Hierbei muss berücksichtigt werden, dass alle Patienten medikamentös behandelt wurden. Für den klinischen Alltag bedeutet dies, dass zunächst bei Patienten mit akuten Rückenschmerzen der Verlauf unter medikamentöser Therapie mit nicht steroidalen Antirheumatika und bei starken Schmerzen mit retardierten Opioiden abgewartet werden kann. Bestehen die Schmerzen länger als 10 bis 14 Tage, sollten dann allerdings Physiotherapie und Krankengymnastik zum Einsatz kommen.

\section{Weitere Infos auf springermedizin.de}

Manuelle Medizin zur Behandlung von Rückenschmerzen Der Artikel diskutiert anhand aktueller Studien die Effektivität manualmedizinischer Verfahren zur Therapie von Rückenschmerzen (6007250). Sie finden ihn, indem Sie den Titel oder die ID-Nummer (in Klammern) in die Suche eingeben.

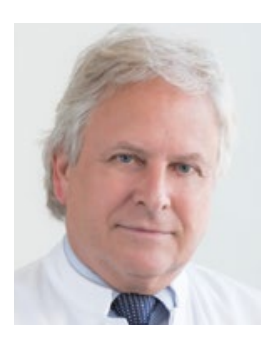

Prof. Dr. med. Hans-Christoph Diener, Essen

Direktor der Klinik für Neurologie, Universitätsklinikum Essen E-Mail:h.diener@uni-essen.de 